\section{Intermittent mydriasis associated with carotid vascular occlusion}

PD Chamberlain ${ }^{1,2}$, A Sadaka $^{2}$, S Berry $^{2}$ and $A G$ Lee $1,2,3,4,5,6$

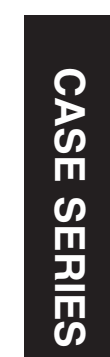

the literature as benign episodic pupillary dilation or BEUM. We report two patients with acquired occlusive disease of the ipsilateral ICA who developed multiple, stereotyped, neurologically isolated, transient episodes of mydriasis consistent with BEUM. We discuss the possible mechanisms, differential diagnosis and recommended evaluation for atypical cases for episodic mydriasis.

\section{Case one}

A 78-year-old man presented with 10 episodes of stereotyped, intermittent, unilateral, painless pupillary dilation of the left eye (OS) lasting minutes to hours at a time without diplopia or ptosis. The first episode happened approximately 1 year prior to presentation. The episodes seemed to be worse in the light. Past medical history was notable for atherosclerotic large vessel, cardiac, valvular, and coronary artery disease treated with coronary bypass grafting, left subclavian bypass, right carotid endarterectomy, cardiac pacemaker placement, and aortic valve replacement. The most recent of these surgeries, aortic valve replacement, took place 1 year prior to the onset of symptoms, but the rest of these surgeries took place 8 or more years prior to onset of symptoms. Because of the extensive vascular disease history, a repeat carotid Doppler was performed and showed complete occlusion of the left ICA. This $100 \%$ occlusion of the left ICA had also been documented by carotid ultrasound 6 months before.

Neuro-ophthalmic exam showed visual acuity of 20/30 in the right eye (OD) and 20/20 in OS. Despite the complaint of BEUM, the pupils, were isocoric under light and dark lighting conditions and were normally reactive to light OU. There was no dilation lag or relative afferent pupillary defect (RAPD) seen. Slit-lamp exam showed no pupil irregularities, vermiform movements, or other iris abnormalities. There was no ptosis or ophthalmoplegia. The remainder of the eye examination was noncontributory. Magnetic
${ }^{1}$ Department of Ophthalmology, Blanton Eye Institute, Houston Methodist Hospital, Houston, TX, USA

${ }^{2}$ Department of Ophthalmology, Baylor College of Medicine, Houston, TX, USA

${ }^{3}$ Departments of Ophthalmology, Neurology, and Neurosurgery, Weill Cornell Medical College, New York, NY, USA

\section{${ }^{4}$ Department of} Ophthalmology, University of Texas Medical Branch, Galveston, TX, USA

${ }^{5}$ Department of Ophthalmology, University of lowa Hospitals and Clinics, Iowa City, IA, USA

${ }^{6}$ Department of Ophthalmology, UT MD Anderson Cancer Center, Houston, TX, USA

Correspondence: AG Lee, Department of Ophthalmology, Blanton Eye Institute, Houston Methodist Hospital, 6560 Fannin Street, Scurlock Tower 450, Houston, TX 77030, USA

Tel: +713 441-8823;

Fax: 713-793-1636.

E-mail: aglee@

houstonmethodist.org

Received: 31 December 2016

Accepted in revised form:

8 June 2017

Published online:

11 August 2017 
resonance (MR) scan and MR angiography of the head and neck showed $100 \%$ occlusion of the left cervical ICA but no other abnormalities. There was no clinical evidence for a Horner syndrome but no topical pharmacologic testing was performed.

\section{Case two}

A 26-year-old female with a history of migraine presented with multiple, recurrent, intermittent, unilateral episodes of painless pupillary dilation OD. These occurred approximately 20 months after sustaining neck trauma by being kicked by a horse. At the time of her initial injury, she suffered a right ICA dissection with secondary 65\% stenosis and was treated with aspirin. In the months following the trauma she suffered a transient left hemiparesis episode and a single 1-min episode of vision loss OD (amaurosis fugax). Cervical CT angiography (CTA) confirmed a right carotid dissection which was stented. She was treated with aspirin, clopidogrel, and apaxiban following a negative cardiac workup for presumed transient ischemic attack and amaurosis fugax OD. Exam showed visual acuity of 20/20 in both eyes and isocoric pupils in the light and in the dark without RAPD. There was no ptosis or evidence of Horner syndrome, and she exhibited full motility. Automated perimetric, slit-lamp, and fundus exams were normal. No topical pharmacologic testing was performed.

\section{Discussion}

BEUM is a relatively common syndrome presumed to be due to transient dysfunction in the pupillary autonomic nervous system. ${ }^{1}$ BEUM typically affects younger women and is often associated with migraine. ${ }^{1}$ No workup or neuroimaging is recommended in typical cases of BEUM. In our two cases, however, atypical features were present that prompted further evaluation. In case one, the patient was an elderly male with no history of migraine and in case two the patient had prior neck and chest trauma and a secondary ICA dissection. A prior case report of intermittent mydriasis in a teenage patient with congenital hypoplasia of the ICA has been described. ${ }^{2}$ Cranial MR imaging of that patient revealed an enlarged posterior communicating artery (PComA) and it was hypothesized that possible intermittent CN III dysfunction was the mechanism. In our case one, we initially hypothesized that perhaps occlusion of the left ICA was associated with secondary enlargement of the PComA which could have produced theoretic compression of the parasympathetic fibers of CN III. Cranial CTA in case one however did not confirm any evidence of PComA enlargement. Thus, it is not clear if sympathetic overaction or parasympathetic underaction is the cause for the transient mydriasis in our case one. In another report, a 37-year-old woman reported two episodes of unilateral mydriasis separated by two years, and was found to have an ipsilateral ICA dissection following workup. ${ }^{3}$ She had a history of severe cervical trauma in a motor vehicle accident several years prior to her first episode, and was suspected to have oculosympathetic spasm as the mechanism (a type of autonomic hyperreflexia associated with cervical spinal cord injury). ${ }^{4}$ It is also possible that surgical manipulation of the great vessels during one of case one's many prior surgeries could also have caused damage to the oculosympathetic pathway with subsequent sympathetic overaction.

In our case two, it is possible that oculosympathetic 'spasm' (overaction) following damage to the sympathetic nerve fibers surrounding the ICA either due to the initial ICA trauma or the subsequent ICA dissection may have been responsible for the intermittent mydriasis. There are also three reports of unilateral mydriasis in the acute setting as the presenting sign of carotid occlusion secondary to dissection, ${ }^{5-7}$ and mydriasis was thought to be secondary to CN III ischemia. None of these previously reported cases displayed intermittent mydriasis. Interestingly, our case two did not report the transient mydriasis episodes at the time of initial injury and the symptoms occurred only later in her course.

Although it is unknown if the episodes in our two cases were causal or coincidental to the ICA disease we propose that transient autonomic dysfunction related to the ICA could account for the intermittent episodes of pupillary dilation. It is not clear if the isolated, episodic mydriasis is due to overaction of the oculosympathetic pathway or underaction of the parasympathetic pathway, but given the ipsilateral ICA disease we suspect the former despite the lack of clinical evidence for oculosympathetic dysfunction (Horner syndrome) clinically. The possibility of acephalgic migraine equivalent in the elderly (case one) or migrainerelated BEUM (case two) cannot be completely excluded.

In summary, transient, neurologically isolated, mydriasis is typically benign (BEUM), but patients who have non-neurologically isolated disease, do not have migraine, are elderly, vasculopathic, or male or who have had recent trauma might be considered for further cranial and neck imaging of the ipsilateral ICA. To our knowledge, these are the first and only such ICAassociated cases of ICA-related transient mydriasis presenting as BEUM in the English language ophthalmic literature. 


\section{Summary}

\section{What was known before}

- Benign episodic unilateral mydriasis is typically reported in young women with a history of migraine and generally does not require any further evaluation or imaging studies.

- One case has previously been reported of a patient with unilateral, painless mydriasis in a patient with ipsilateral congenital hypoplasia of the internal carotid artery. This was thought to be caused by intermittent compression of cranial nerve III secondary to posterior communicating artery enlargement.

\section{What this study adds}

- This study presents two cases of episodic, unilateral, painless mydriasis in patients with acquired internal carotid artery occlusive disease and no evidence of posterior communicating artery enlargement.

- We hypothesize that autonomic nervous system dysfunction related to the internal carotid artery occlusion or disruption of the oculosympathetic pathway with subsequent aberrant regeneration following trauma or surgery accounts for these episodes in our patients.

- Although further evaluation and imaging are not typically recommended for BEUM, atypical cases (for example, elderly, vasculopathic, male, or history of trauma) of episodic unilateral mydriasis may benefit from imaging of the ipsilateral ICA.

\section{Conflict of interest}

The authors declare no conflict of interest.

\section{References}

1 Jacobson DM. Benign episodic unilateral mydriasis. Ophthalmology 1995; 102(11): 1623-1627.

2 Ibrahim M, Branson HM, Buncic JR, Shroff MM. A case of Horner syndrome with intermittent mydriasis in a patient with hypoplasia of the internal carotid artery. AJNR Am J Neuroradiol 2006; 27(6): 1318-1320.

3 Orssaud C, Roche O, Renard G, Dufier JL. Carotid artery dissection revealed by an oculosympathetic spasm. J Emerg Med 2010; 39(5): 586-588.

4 Kline LB, McCluer SM, Bonikowski FP. Oculosympathetic spasm with cervical spinal cord injury. Arch Neurol 1984; 41(1): 61-64.

5 Sharma GK, Deshmukh VR, Albuquerque FC, Wolf TR, McDougall CG. Resolution of mydriatic pupil after angioplasty and stenting of cervical internal carotid artery dissection: case report. Neurosurgery 2009; 64(3): E562-E563.

6 Inzelberg R, Nisipeanu P, Blumen SC, Kovach I, Groisman GM, Carasso RL. Transient unilateral mydriasis as the presenting sign of aortic and carotid dissection. Neurology 2000; 55(12): 1934-1935.

7 Koennecke H, Seyfert S. Mydriatic pupil as the presenting sign of common carotid artery dissection. Stroke 1998; 29(12): 2653-2655. 